\title{
What could the LHC teach us on the structure of space-time?
}

\author{
George Triantaphyllou, a \\ ${ }^{1}$ National Technical University of Athens \\ 58 SINA Str., GR 10672 ATHENS, Greece
}

\begin{abstract}
Collision energies of proton beams now available at the LHC increase the probability of discovering the inner works of the Brout-Englert-Higgs (BEH) mechanism within the foreseeable future. Nevertheless, they are still several orders of magnitude below the scale where a possible non-trivial structure of space-time would be detectable. Apart from remaining completely silent on the issue of the fundamental nature of elementary particles and the space in which they propagate, one may try to speculate on this matter by carefully extrapolating existing scientific methods and knowledge to Planck energies. In this talk, an effort is made to logically link some potential discoveries at the LHC with specific space-time structures. Since such links are inevitably weak due to the huge energy hierarchy between the electro-weak and the Planck scales, our goal does not exceed a mere presentation of naturalness and self-consistency arguments in favor of some of the possible outcomes, placing particular emphasis on the scenario of the mirror world.
\end{abstract}

\section{Introduction}

After several decades of efforts both in the experimental and in the theoretical front, high-energy physicists are awaiting for fresh collision data from the upgraded LHC which could potentially shed more light on the origin of electro-weak symmetry breaking. The energies of the proton beams increase the probability of discovering new physics beyond the Standard Model (SM) which might lie behind the BEH mechanism. Even though no significant deviations from the Glashow-WeinbergSalam model containing just an elementary Higgs field have been detected so far, it is well known that the mass of such a field is unstable against quantum corrections. If nothing new is introduced to stabilize its mass, not only would it acquire a Planck-scale mass, but it would also render all other known particles much heavier than they are found to be. This instability reminds us that when a theory attempts to introduce an arbitrary hierarchy between energy scales with no special reason, nature has a way, of quantum mechanical origin, to eliminate this unnaturalness. There have been several proposals over the years trying to explain the stabilization of the electro-weak scale to such small values compared to the scale where gravity effects might set in, altering probably in a fundamental way the inner structure of space-time. Some of them, apart from introducing new fields or phenomena at energies in principle accessible at the LHC, allow us to speculate also on what general form such alterations might take.

a e-mail: gtriantaphyllou@aya.yale.edu 
In the following, we choose to discuss some of these alternatives, one more extensively than the others since it has not been studied as extensively as the others, which could potentially hide behind the BEH mechanism, and which have already started being under scrutiny by LHC data. The first introduces large extra dimensions, the second introduces supersymmetric particles, and the third mirror particles, all expected near the $\mathrm{TeV}$ scale which is relevant to the $\mathrm{BEH}$ mechanism. This choice is obviously not exhaustive, since it is always possible that Nature keeps something else in store for us, but it is mainly guided by the characteristic differences of their implications on the structure of space-time. The huge hierarchy between the electro-weak and the Planck energy scales render the ground on which such speculations reside, and especially attempts to establish a relevant "one-to-one" correspondence, shaky. Nevertheless, the form which the stabilizing mechanism will be hopefully found to take is one of our last chances for several decades to come, at least regarding accelerator experiments, to constitute a basis of a guess concerning the true nature of elementary particles, their interactions and space-time. This is also due to the fact that the proposals discussed here are compatible with the "desert hypothesis", introducing new Physics around the scale of $1 \mathrm{TeV}$ and then nothing else until scales within 1-3 orders of magnitude away from the Planck scale. This situation highlights the critical conjuncture of present-day high-energy Physics in an effort to discover the fundamental principles of Nature. Therefore, although the final results should be taken cum grano salis, the importance of the questions involved render the relevant theoretical speculations valuable.

\section{Phenomenological inductive approach}

\subsection{Potential implications}

One very interesting alternative which could stabilize the electro-weak scale is to assume that there are extra spacial dimensions compactified on a radius relevant to the BEH mechanism [1]. This eliminates by "brute force" the hierarchy between the Planck scale and the electro-weak scale by a radical alteration of the structure of space-time at scales which could be in principle accessible at the LHC, or the colliders next to it, by the production of mini black-holes and other phenomena [2]. Even though models with large extra dimensions are usually confronted with problems related to the electron magnetic moment which is measured experimentally very precisely, they offer a typical example of a way by which the $\mathrm{BEH}$ mechanism could signal a very important shift of our understanding of the nature of space-time. Recent experimental bounds on such models are found in [3].

Another alternative which has been particularly popular during the last decades is to assume that the known elementary particles have "supersymmetric partners", i.e. fields with the same gauge charges but spins differing by one half unit from the spins of the original particles. The existence of such hypothetical particles leads to the cancellation of quantum corrections which destabilize the mass of elementary Higgs scalars, offering thus an effective solution to the hierarchy problem. In this scenario, the required stabilization is due to a new space-time symmetry called "supersymmetry" which accounts for the existence of these new "partners". These new elementary particles can be studied perturbatively since they are weakly coupled at LHC energies. Moreover, they may lead to a unification of the gauge-coupling strengths at much higher energies [4], which would not only imply that proton decay should be detectable in future experiments but also that a unifying theory can incorporate all this rich structure of fields in a symmetric way. In this respect, the new space-time symmetry proposed, apart from solving the hierarchy problem, is an important ingredient in attempts unifying gravitational interactions with the other known gauge interactions in the context of string theory [5].

According to this theory, elementary particles at Planck energies can be considered as strings propagating perturbatively on a continuous space-time background. Moreover, extra space dimensions are 
expected to influence directly all relevant physical considerations, allowing membrane solutions with dimensionality higher than the one of strings [6]. From such a view point, it is apparent that a supersymmetric solution to the hierarchy problem might also signal a radical shift of our understanding of the fundamental nature of particles and space-time. Even thought the "stringy" nature of particles is not expected to be detectable at the LHC, unless some of the extra dimensions are large, discovering supersymmetric particles would offer a mild indication that the unification program leading to string theory is in the right direction. Although it would be far from being a proof of the "stringy" nature of elementary particles and of extra dimensions at Planck energies, it would at least fulfill the minimal requirement of self-consistency, which, due to the importance and difficulty of the problems at hand, is not a trivial achievement. Constraints on relevant models can be found in [7].

Last but not least, we come to the third alternative, which will be mostly discussed here, according to which known fermions have mirror "partners" usually called "katoptrons". Contrary to considering fermions having zero-spin "partners", katoptron theory introduces new fermion fields with opposite chirality from the known fermions. Apart from carrying the same known gauge charges, katoptrons interact with each other via a new gauge interaction described by an SU(3) mirror-family symmetry. This interaction becomes naturally strong at around $1 \mathrm{TeV}$ and breaks the electro-weak symmetry dynamically. In this scenario, the BEH mechanism is an effective description of a strongly-coupled theory similar to QCD, where instead of elementary Higgs fields or free mirror fermions, one expects several bosonic bound states, i.e. spin-0 and spin-1 mirror mesons which might show up in the ATLAS and CMS experiments at the LHC. Stabilization of the electro-weak scale is achieved by the compositeness of the Higgs fields, which can be traced back to a gauge symmetry.

Mirror fermions were first proposed in the Lee-Yang locus classicus on parity-symmetry violation [8] and were later used in [9] in order to break the electro-weak symmetry dynamically via mirrorfermion condensates. This was proposed in a spirit similar to [10], which in its turn was inspired by the fermion condensates appearing in BCS superconductivity [11]. Using a new gauge interaction felt only by katoptrons and becoming strong around $1 \mathrm{TeV}$ assuming unification of all gauge-coupling strengths around the Planck energy was first achieved in [12]-[13], in continuation of a long tradition starting from Pati-Salam models, freeing the theory from Landau poles and completing it grouptheoretically [14]. Similarly to supersymmetric theories, providing a unifying framework not only leads to a prediction of proton decay, but also enables unification of all interactions including gravity, albeit in a context distinct from string theory. In fact, considering elementary particles as vacancies within an emergent dynamical discrete space-time leads to models incorporating mirror fermions [13]. Therefore, detection of mirror mesons or other katoptron effects at the LHC, far from being a direct probe of Planck-scale Physics, could be considered as a possible indication of, or even more conservatively just as consistent with, some kind of space-time discreteness. Before returning to this important issue more extensively in the next section, we briefly review some of the possible relevant signals accessible at the LHC.

\subsection{Mirror mesons and their decays}

A classical study of hadron-collider phenomenology of models based on strongly-interacting fermions, which initially went under the name of "technicolor", is [15]. The relevant program here consists of studying similar production and decay mechanisms of mesons formed as bound states of the hypothetical fermions introduced, since confinement is not expected to allow katoptrons to propagate freely in space. Therefore, the focus of LHC experiments trying to test such theories should be on excesses of di-boson and di-fermion production originating from new intermediate states analogous to QCD hadrons having either zero spin, i.e. mirror scalar and pseudo-scalar mesons like pions, or spin-1, i.e. mirror vector mesons like rhos. Non-perturbative dynamics do not allow a detailed 
quantitative study of the relevant cross-sections as in weakly-coupled theories. Nevertheless, it is still possible to make some general qualitative remarks on what should be generally expected.

We first note that SM quantum numbers for mirror fermions give rise to a mirror chiral symmetry $\mathrm{SU}(8)_{L} \times \mathrm{SU}(8)_{R}$ subsequently broken by the strong coupling of the mirror family group SU(3) down to a diagonal vector $\mathrm{SU}(8)$ flavor symmetry corresponding to four isospin doublets, i.e. one color-singlet and one color-triplet, analogous to Gell-Mann's flavor symmetry in QCD. This leads to a mirror meson spectrum corresponding to the broken generators of the axial SU(8) symmetry, thus including, apart from the resonances $\sigma^{K}, \eta^{K}$ and $\omega^{K}$, with the superscript $K$ a reminder of their katoptron content, analogous to the scalar $\sigma$, pseudo-scalar $\eta$ and vector $\omega$ mesons in QCD, sixty-three (pseudo-scalar) mirror pions $\pi^{K}$, along with sixty-three (spin-1) mirror $\rho \mathrm{s}, \rho^{K}$ having the same quantum numbers except from spin, their number being equal to the dimension of the adjoint representation of the flavor group. Furthermore, one can not exclude the existence of the corresponding parity-even and heavier excited states of these mesons.

In this scenario, the $\sigma^{K}$ meson has quantum numbers equal to the SM Higgs particle, while three mirror pions, $\pi^{K} 20$ and $\pi^{K 2 \pm}$, are "eaten" by the electro-weak gauge bosons, becoming thus the longitudinal components of $W^{ \pm}$and $Z^{0}$. This leaves us with five remaining color-singlet pseudoscalars, i.e. three electrically neutral along with one positively and one negatively charged, from the initially eight color-singlet mirror pseudo-scalar mesons, in addition to eight color-singlet mirror vector mesons:

$$
\pi^{K 10}, \pi^{K 1 \pm}, \pi^{K 10 \prime}, \eta^{K}(\text { spin - } 0) \text { and } \rho^{K 1,20}, \rho^{K 1,2 \pm}, \rho^{K 0 \prime} \text { and } \omega^{K} \quad(\text { spin - 1). }
$$

Regarding the rest of the mirror mesons, eight of each kind are color-triplets denoted by

$$
\pi_{3}^{K 1,2,21,5}, \bar{\pi}_{3}^{K 1,2,2 \prime, 5}(\operatorname{spin}-0) \text { and } \rho_{3}^{K 1,2,21,5}, \bar{\rho}_{3}^{K 1,2,21,5}(\operatorname{spin}-1) \text {, }
$$

all of them fractionally charged (either $-\frac{1}{3}$ for $\pi_{3}^{K}{ }^{1}, \rho_{3}^{K}{ }^{1}$, or $\frac{2}{3}$ for $\pi_{3}^{K 2,2 \prime}, \rho_{3}^{K 2,2 \prime}$, or $\frac{5}{3}$ for $\pi_{3}^{K}{ }^{5}, \rho_{3}^{K 5}$ ) and usually called "lepto-quarks", and four of each kind are color-octets, denoted by

$$
\pi_{8}^{K 0}, \pi_{8}^{K \pm}, \pi_{8}^{K 0 \prime} \quad\left(\text { spin - 0) and } \rho_{8}^{K 0}, \rho_{8}^{K \pm}, \rho_{8}^{K 0 \prime} \quad(\text { spin - 1) }\right.
$$

respectively [15]. Note that isospin-singlet mesons are denoted either by $\sigma^{K}, \eta^{K}, \omega^{K}$ or by primed symbols above, the rest being members of isospin triplets. Another obvious fact to bear in mind is that mirror mesons with equal charge and color may mix with each other, like $\pi^{K 10}$ with $\pi^{K 10}, \pi_{3}^{K 2}$ with $\pi_{3}^{K 2 \prime}, \pi_{8}^{K} 0$ with $\pi_{8}^{K}{ }^{0 \prime}$, and similarly for their vector-meson counterparts.

The masses of these mesons are not easy to estimate, and large-N arguments of [15] are questionable due to the fact that the katoptron family group becomes strongly coupled and breaks at energies $\Lambda_{K} \sim$ $1 \mathrm{TeV}$ from SU(3) down to $\mathrm{SU}(2)$. Therefore, one expects a doubling of the meson mass spectrum due to the hierarchy, denoted by $r$, of the corresponding energy scales of mirror-fermion chiral symmetry breaking, or even its tripling according to the extent by which the breaking of the remaining SU(2) family symmetry affects the corresponding mirror-meson masses. Estimating this hierarchy yields a factor $r$ of around

$$
r \sim \exp \left(C_{2}(S U(3))-C_{2}(S U(2))\right) \sim 5.75
$$

with $C_{2}(g)$ the quadratic Casimir invariant of a Lie algebra $g$ [16], noting that $r \sim 5$ is easier reconcilable with the weak scale and with present experimental bounds. Therefore, the two lighter mirror generations are expected to have masses of around $\Lambda_{K} / r=200 \mathrm{GeV}$. In the following, the lighter mirror mesons, denoted by $\pi_{A}^{K}$ and $\rho_{A}^{K}$, omitting numerical superscripts and color subscripts, correspond to katoptrons with masses $M_{A} \sim 200-300 \mathrm{GeV}$, bearing in mind that they might be further split into two 
distinct subgroups according to the mass of their mirror-fermion content. Similarly, the heavier mirror mesons are denoted by $\pi_{B}^{K}$ and $\rho_{B}^{K}$, corresponding to katoptrons masses $M_{B} \sim \Lambda_{K} \sim 1-1.5 \mathrm{TeV}$.

Allowing a slight digression, note that the mass hierarchy between third-generation SM quarks and the other fermion generations can be traced back to the hierarchy of mirror-fermion chiral-symmetrybreaking scales [16]. This happens because the breaking of the mirror-family symmetry allows the quantum-mechanical mixing of mirror fermions with the known fermion fields, providing thus a mass "feed-down" mechanism which is free not only of flavor-changing-neutral currents, but also of toolight pseudo-Goldstone bosons and of a too-large $\Delta \rho$ parameter [17], all problems usually confronting extended-technicolor (ETC) models. Furthermore, it might provide for deviations from QCD-like strong dynamics accommodating a small $S$ parameter, possibly related to a reduced or even inverted mass hierarchy between vector and axial-vector mirror mesons. In addition, the generalized mass matrix mixing mirror- with ordinary fermions provides an origin not only for the Cabibbo-KobayashiMaskawa (CKM) matrix but also for small neutrino masses via a see-saw mechanism [12]. Moreover, this framework allows the introduction of complex phases violating the weak CP symmetry potentially leading to the observed baryon asymmetry of the universe. Last, the simultaneous existence of two groups of light and heavy mesons is not only a by-product of the breaking of the mirror-family group, but it is also required for self-consistency, since mesons only as light as the recently discovered Higgslike scalar would be hardly able to reproduce the electro-weak scale correctly [16].

To return to the issue at hand, order-of-magnitude estimates based on QCD for mirror mesons would give masses of around $2 M_{A} \sim 400-600 \mathrm{GeV}$ for the lighter $\eta_{A}^{K}, \rho_{A}^{K}$ and $\omega_{A}^{K}$ and $2 M_{B} \sim 2-3 \mathrm{TeV}$ for the heavier $\eta_{B}^{K}, \rho_{B}^{K}$ and $\omega_{B}^{K}$. On the contrary, mirror pions should be relatively light if considered as Nambu-Goldstone bosons of the broken mirror chiral symmetry. However, since the gauged mirror family symmetry is consecutively broken at around $1 \mathrm{TeV}$ and $170 \mathrm{GeV}$, mirror-pion and $\sigma^{K}$ masses are also expected to be grouped either around $M_{A} / 2 \sim 100-150 \mathrm{GeV}\left(\sigma_{A}^{K}, \pi_{A}^{K}\right)$ or around $M_{B} / 2 \sim 0.5$ $0.75 \mathrm{TeV}\left(\sigma_{B}^{K}, \pi_{B}^{K}\right)$. Note that colored mirror pions receive additional contributions to their masses due to QCD, which would be on the order of $\sqrt{\alpha_{s}} M_{\rho^{K}} \sim 200-300 \mathrm{GeV}$ were the katoptron-family group to remain unbroken and have a single scale $M_{\rho^{K}}$ for $\rho^{K}$ masses, with $\alpha_{s}$ the value of the QCD coupling at the mirror pion mass. The breaking of the family symmetry implies however that these contributions might lie in the range of $0.1-0.8 \mathrm{TeV}$ depending on the mass-spectrum group to which the colored meson belongs. In this picture, the meson $\sigma_{A}^{K}$ corresponds to the "Higgs-like" particle detected at the LHC, in analogy to QCD where the lowest-lying resonance heavier than the three pseudo-scalar pions, the analogues of which are here "eaten" by $W^{ \pm}$and $Z^{0}$, is the scalar meson $\sigma$. The fact that the mass of the scalar particle detected is lower than double the masses not only of the electro-weak gauge bosons but of the top-quark as well might partially explain its relatively small width compared to the one of the sigma meson in QCD, which mainly decays into two pions.

In any case, the dynamics involved are so complicated that one should consider the mass ranges above with a fair amount of flexibility, which should be reflected in the relevant computer simulations and in the LHC data analysis. In fact, due to the breaking of the mirror family symmetry, mirror fermions might not have enough time to hadronize, either decaying before meson formation or forming very broad resonances. Assuming that mirror mesons do form, the discussion above implies a potentially very rich phenomenology which should show up in particle excesses in several processes [18]. Given precise experimental constraints, one should aim at characteristic signals which not only are more probable to be distinguished from background but also which can be distinguished from similar signals predicted by alternative theories. First of all, mirror meson phenomenology seems similar to what is expected in ETC scenaria following [19]. Therefore, only by focusing on the distinctive features of katoptron theory may one be able to favor one model over the other. Since the katoptron mass feed-down mechanism is based on quantum mixing instead of ETC interactions, the relevant effects 
on the heavier fermions like the top quark should be more pronounced. In particular, the CKM matrix element $\left|V_{t b}\right|$ is expected to deviate from unity by about $5 \%$. In addition, deviations from the SM leftand right-handed weak couplings of top quark should show up in conveniently-chosen asymmetries. Last, at much higher energies where katoptrons are no longer interacting strongly with each-other, or in the case where katoptrons do not hadronize, a forward-backward asymmetry of mirror fermions opposite to the one expected from SM fermions, or similar deviations from standard left-right weakcharge assignment, should be detectable. Given that such measurements require precision that can be easier achieved by a high-energy (3-4 TeV [18]) electron collider like CLIC and ILC or a muon collider rather than a hadronic collider, one should seek other methods of signal differentiation according to each model. One important difference for instance is the breaking of the katoptron-family gauge group, which, apart from rendering katoptrons unstable and unlikely to be connected to Dark Matter (DM) particles, leads to the lifting of the degeneracy of the mirror meson spectrum, something not expected in usual technicolor models. This leads to some relatively heavier pseudo-scalar mirrormeson masses (belonging to the "B" group) and some relatively lighter vector mirror-meson masses (belonging to the "A" group) than in typical technicolor models. This implies the prediction of many more resonances, but it changes the luminosities needed to detect their decays over background, since cross-sections depend not only on the new meson masses but also on the size of the new -unbrokenstrong gauge group [15]. Taking the scalar meson $\sigma_{A}^{K}$ as an example, its decay to two photons via new heavy-fermion triangle Feynman diagrams depends quadratically on the number of "colors" of the new strong interaction. Therefore, lack of observation of large deviations of the two-photon Higgs decay width from its SM value is consistent with the breaking of the mirror family group, possibly indicating even a tripling of the mirror-meson mass spectrum. We discuss in the following some processes of potential interest for the LHC assuming just a doubling of the mass spectrum. According to similar analyses [15][18][20], promising candidates detectable over background with the least integrated luminosity are the decays of the neutral color-octet pseudo-scalar mirror pions, $\pi_{8}^{K}{ }_{A, B}$, produced mainly by gluon fusion but also quark-anti-quark annihilation, to a top-anti-top quark pair.

The special interest in this decay is due to the fact that mirror-pion decay widths $\Gamma\left(\pi^{K} \longrightarrow \bar{f}_{i} f_{j}\right)$ stemming from Yukawa interactions depend quadratically on the final-fermion masses $m_{i, j}$ :

$$
\Gamma\left(\pi_{8 A, B}^{K} \longrightarrow \bar{f}_{i} f_{j}\right) \sim \Gamma\left(\pi_{3 A, B}^{K} \longrightarrow \bar{f}_{i} f_{j}\right) \sim \frac{G_{F} p\left(m_{i}^{2}+m_{j}^{2}\right)}{16 \pi} \sim p\left(\left(m_{i} / 2.1 \mathrm{TeV}\right)^{2}+\left(m_{j} / 2.1 \mathrm{TeV}\right)^{2}\right),
$$

where $G_{F}$ is the Fermi constant and $p$ is the fermion momentum in the mirror-pion rest frame. Although factors depending on the size of the new gauge group lead to a relative enhancement of the production mechanism in usual technicolor models, in katoptron models their effect is questionable due to the breaking of the SU(3) mirror-family group [18]. The main background to this process comes from QCD, which can be significantly reduced via stringent rapidity cuts since, contrary to this background, mirror-pion decay products are expected to be spherically distributed. Furthermore, the lighter mirror pion $\pi_{8 \mathrm{~A}}^{K}{ }_{\mathrm{A}}^{\prime}$ should be easier detectable due to the fall of cross sections with energy.

Nevertheless, there are two problems apart from the large QCD background rendering the observability of this process questionable. First, the pion mass should preferably be larger than twice the mass of the top quark, i.e. larger than about $350 \mathrm{GeV}$. This exceeds our previous estimates for the masses of the lighter mirror pions, even though it cannot be completely ruled out beforehand. Second, the breaking of the mirror-family symmetry leads to mixing of ordinary fermions with their mirror partners favoring processes which respect fermion generations [16]. Therefore, "group B" mirror scalar and pseudoscalar mesons are expected to mainly couple to "group B" mirror vector mesons and third-generation ordinary fermions, while "group A" mirror scalars and pseudo-scalars are expected to mainly couple to "group A" mirror vector mesons and first- and second-generation ordinary fermions. Consequently, 
the decay of $\pi_{8 A}^{K}{ }_{A}^{\prime \prime}$ to a bottom-anti-bottom quark pair, even when kinematically allowed, is expected to be suppressed due to the small mixing between first- and second-generation katoptrons with thirdgeneration SM fermions. Processes of main interest are thus "generation-respecting", allowing obvious extension of this argument to the aforementioned possible tripling of the mirror-meson mass spectrum. Therefore, heavier color-octet mirror pions, expected to have masses well over $1 \mathrm{TeV}$, i.e. large enough for on-shell production of top quarks, are expected to couple to third-generation fermions, leading to:

$$
g g, \bar{q} q \longrightarrow \pi_{8 B}^{K 0 \prime} \longrightarrow \bar{t} t \text { or } \bar{b} b .
$$

However, since cross-sections decrease with energy quite fast, the signal needed to exceed the relevant SM background requires much higher integrated luminosities than the ones estimated for lighter mirror mesons, unless $\pi_{8 B}^{K 0}$ f finally turns out to be much lighter, and this process' advantage over alternative ones becomes questionable. Other processes of interest are the decays of heavy neutral color-singlet mirror spin-0 mesons to a $\tau \bar{\tau}$ or a diphoton pair: $g g \longrightarrow \sigma_{B}^{K}$ or $\pi_{B}^{K 1}{ }^{\prime \prime} \longrightarrow \bar{\tau} \tau$ or $\gamma \gamma$, enjoying the advantage of being QCD-background free. Nevertheless, since the cross-sections in the difermion case depend on the masses of the final fermions and in the diboson case correspond to a higher-loop process, $b$-quark, $\tau$-lepton and $\gamma \gamma$ final states have a disadvantage when compared to $\bar{t} t$ pairs ceteris paribus.

A different promising process is related to the pair production of color-octet mirror pions $\pi_{8}^{K}$ via $\bar{q} q$ annihilation and gluon fusion, which can be further enhanced by intermediate mirror vector mesons. Take as an example the following generation-respecting processes:

$$
\begin{aligned}
& g g \quad \longrightarrow \quad \text { direct, } g, \rho_{8 A}^{K 0 \prime} \longrightarrow \pi_{8 A}^{K+} \pi_{8 A}^{K-} \longrightarrow \bar{s} c+\bar{c} s \\
& g g \longrightarrow \text { direct, } g, \rho_{8 B}^{K 0} \longrightarrow \pi_{8 B}^{K+} \pi_{8 B}^{K-} \longrightarrow \bar{b} t+\bar{t} b,
\end{aligned}
$$

where "direct" denotes either t- and u-channels or absence of any s-channel intermediate state, and symbols on either side of "commas" refer to processes interfering with each other. The quarks produced are generally not collinear with their anti-particles, leading to signals which may in principle be distinguished from QCD background via appropriate cuts. Note however that it might be difficult to distinguish the signals discussed so far from similar processes originating from elementary scalars predicted by supersymmetric theories [15]. This regards especially the $\sigma_{A}^{K}$ and $\pi_{A}^{K}$ mirror mesons, since $\pi_{B}^{K}$ mirror mesons have masses generally too heavy for supersymmetry, and mirror vector mesons have spin incompatible with the fields introduced by usual supersymmetric models. Another process of interest is the decay of charged mirror vector mesons, produced by interactions between up- and down- quarks $q_{i}$ and $q_{j}$, to an electro-weak gauge boson pair, in analogy to [15]:

$$
\bar{q}_{i} q_{j} \longrightarrow \rho_{A, B}^{K 2} \longrightarrow W^{ \pm}+Z^{0} .
$$

Recent event excesses at around $2 \mathrm{TeV}$ at the LHC [21] might be related to a process involving $\rho_{B}^{K 20, \pm}$ or to heavier excitations of these resonances. Nonetheless, models with extra massive vector gauge bosons mimic the mirror-meson effects of this process. Therefore, such enhancements should be considered in conjunction with other effects in order to draw safer conclusions on the theory behind them.

The problem of signal ambiguity is not so acute in the case of a set of quite interesting processes regarding the pair production of lepto-quarks $\pi_{3}^{K}$. Lepto-quarks can be pair-produced via quark-antiquark annihilation and gluon fusion, a process which can be similarly further enhanced by intermediate mirror vector mesons. Since lepto-quarks are expected to decay predominantly into one heavier quark and one heavier lepton, they are expected to lead to characteristic signatures at the LHC. For 
instance, generation-respecting processes like

$$
\begin{aligned}
& g g \longrightarrow \text { direct, } g, \rho_{8 A}^{K 0 \prime} \longrightarrow \pi_{3 A}^{K 5} \bar{\pi}_{3 A}^{K 5} \longrightarrow \bar{\mu} c+\bar{c} \mu \text { or } \bar{e} u+\bar{u} e \\
& g g \longrightarrow \text { direct, } g, \rho_{8 B}^{K 0 \prime} \longrightarrow \pi_{3 B}^{K 5} \bar{\pi}_{3 B}^{K 5} \longrightarrow \bar{\tau} t+\bar{t} \tau \\
& g g \longrightarrow \text { direct, } g, \rho_{8 B}^{K 0 \prime} \longrightarrow \pi_{3 B}^{K}{ }_{B}^{2} \bar{\pi}_{3 B}^{K 2} \longrightarrow \bar{v}_{\tau} t+\bar{t} v_{\tau} \text { or } \bar{\tau} b+\bar{b} \tau \text {, }
\end{aligned}
$$

where the quarks and the leptons are generally expected to be non-collinear with their anti-particles, may lead to signals which can be in principle distinguished from background via appropriate directional cuts. Furthermore, note that the quadratic dependence of the lepto-quark decay width on the final-fermion masses renders it so small for all SM fermions except for the top quark, that present experimental lower bounds on lepto-quark masses can be improved considerably [22].

In any case, the number of detected mirror mesons and the luminosities required to have clear signals based on excess production of heavy fermion flavors depend crucially, apart from the widths of the new resonances, on the experimental resolution in invariant mass and direction of the reconstructed decay channels. Furthermore, of interest might be rarer processes involving $\rho_{3}^{K}$ vector mesons, general $\pi_{B}^{K}$ or $\rho_{B}^{K}$ decays down to $\pi_{A}^{K}$ meson pairs, and heavier mirror hadrons possibly existing which are singlets under the mirror-family group and involve three katoptrons (like mirror protons $p^{K}$ and mirror neutrons $n^{K}$ ), or even four and five katoptrons (analogous to QCD tetra- and penta-quarks). Moreover, one should study processes possibly violating lepton universality like leptonic $B$-meson decays due to the preferential coupling of katoptrons to heavier fermions. Note that the list of processes presented here is not exhaustive, nature being able to reveal its secrets in high-energy experiments in ways which are not a priori considered as the most probable. In any case, computer simulations of the processes above would give us a better picture of what to expect at the RUN II of the LHC.

\section{Analytical deductive approach}

\subsection{Space-time structure}

Supposing that signals like the above do appear, what would be the implications on our understanding of space-time? Taking an opposite direction from [13], we start here from first principles and via a top-down approach end up with a picture consistent with potential experimental findings. Although going from higher to lower energies might appear in certain aspects as non-rigorous and speculative, it facilitates focusing on various open issues to be resolved later. In the case of large extra dimensions and string theory, one assumes a continuous space-time background on which various fields propagate. The size and symmetry of these dimensions is left to be determined, while the internal symmetries are chosen in a self-consistent, ad hoc manner. On the other hand, katoptron theory introduces the "optimal connectivity" principle, with the fundamental degrees of freedom a multiset of identical, distinct elementary entities ("nodes") connected to each other optimally. Connections between members of this multiset, considered as identical Platonic copies of a single Parmenidean archetypical entity suited for an axiomatic formulation, lead to the emergence of space-time and elementary-particle symmetries. Each link connecting two nodes is associated with $\lambda$ (or $J$ in [13]) corresponding to a negative energy connected to energy-minimization arguments and proportional to the cosmological constant. The system grows by gradually connecting more nodes forming a complex network as a discrete space-time where points are replaced by nodes. The number of links of each node is not specified a priori but is determined by the dynamics. The system's self-organized criticality leads to optimal configurations, which are usually laminated lattices.

Considering only a finite number of connections for each node complies with Pythagorean parsimony and is much more, not less, conservative than assuming that each space-time point has an uncountably 
infinite number of nearest neighbors, as implied by space-time continuum. Elementary particles appear here as finitely-extended (in agreement with Aristotle's arguments) topological defects coming in finite species (analogous to Empedocles' and Plato's finite classification of elements, contrary to Democritus' infinite species of "atoms" of various colors and shapes), like lattice vacancies within a discrete, dynamical space-time forming a dendrite, a "world crystal" [23]. This approach, reminiscent of Plotinus views on matter which, due to its variability, cannot correspond directly to a truly fundamental essence, introduces a duality between space-time and matter which may be also regarded as an intriguing instance of a yin-yang dialectic connection. This picture might also address non-locality issues in quantum mechanics, since one or a few missing connections between defects with a common origin spatially receding from each other, which in a dual picture leads to quantum entanglement, explains the long-range correlations needed to interpret phenomena like the EPR paradox.

We study qualitatively this network via the $q=1$ Potts Hamiltonian $H_{P}=-\lambda \sum_{<i, j>} \delta\left(s_{i}, s_{j}\right)$ generally used to analyze disorder-order phenomena like percolation, spin-glass transitions, and even cognition modeling, the sum over the nodes $i, j$ being over nearest neighbors, $s_{i, j}=0$ or $1, \lambda>0$ the coupling strength and $\delta\left(s_{i}, s_{j}\right)=1$ when $s_{i}=s_{j}=1$, being zero otherwise. This system of nodes modeling the "pre-bib-bang" world where usual interactions and space-time have not yet emerged lies initially in a highly-probable state having large temperature $T$ and entropy, without need for fine-tuned cosmological initial conditions. Below the critical temperature $T_{c}=\lambda /\left(k_{B} \ln 2\right)$ however, the system forms configurations based on sphere packings maximizing the Newton (kissing) number and leading to optimal information propagation. For $d=7$, this yields the $E_{7}$ root lattice formed by the unit-norm imaginary Cayley octonions, and for $d=8$, the root lattice of $E_{8}$ (both exceptional symmetries used in particle Physics since [24]), which is even and self-dual, a property crucial for consistent quantization. The $E_{8}$ lattice is more stable than all lower- $d$ and known higher- $d$ configurations at least up to the optimal Leech lattice in $d=24$, while known optimal sphere packings for $8<d<16$ are non-lattice, and for $d>8$ they do not correspond to roots of a Lie-group. Moreover, the asymptotic behavior of the potential $\mathcal{V}(d)=-\lambda \phi(d) K(d)$ for large $d$, with $K(d)$ the maximum possible (instead of using the maximum known in $\mathcal{V}_{\text {opt }}[13]$ ) kissing number of a $d$-dimensional sphere packing and $\phi(d)$ its density, after using the Kabatianskii-Levenshtein bounds [25] for $\phi(d) \stackrel{d \rightarrow \infty}{\longrightarrow} 2^{\alpha_{\phi} d}$ and $K(d) \stackrel{d \rightarrow \infty}{\longrightarrow} 2^{\alpha_{K} d}$, is

$$
\mathcal{V}(d, \lambda=1) \stackrel{d \rightarrow \infty}{\longrightarrow}-2^{\alpha d} \rightarrow 0^{-},
$$

where $\alpha \equiv \alpha_{\phi}+\alpha_{K}=-0.599+0.401 \sim-0.2$, implying stability of the system only for $d \stackrel{\sim}{<} d_{\max } \sim 100$ (a very rough estimate, a challenge for future computations being to possibly exclude potential minima other than the actually known $\mathcal{V}_{\text {actual }}\left(d=\left\{\begin{array}{c}8 \\ 24\end{array}\right\}, \lambda=1\right) \sim\left\{\begin{array}{c}-60 \\ -394\end{array}\right\}$, with $\mathcal{V}_{\text {actual }}(d=48, \lambda=1) \sim-1$ for instance not proven to be optimal for $d=48$ ) and preference for discreteness. A higher- $d$ analogue of spin-glass phase transitions explains thus the emergence of a lattice $L_{G}$ based on the roots of $G \equiv E_{8} \times E_{8}^{\prime}$ at the beginning of our Universe [13], as a "liquid-to-solid" (freezing), "glass-to-crystal" transition. Information and coding theory employ $L_{G}$ also, suggesting the use of a fuzzy version of node multisets as a quantum-computer model of the Universe reminiscent of the Ulam-von Neumann cellular automata. Furthermore, the complexity of this lattice indicates that attempts to consider particles as extended objects or to discretize space need several new dimensions.

Cosmological implications of the above include [13]: (a) Bursts of $\gamma$-rays originating from transitions of metastable "world-crystal" regions to stabler ones; (b) structure formation and intergalactic voids considered as regions with distinct lattice topologies emerging from initial quantum inhomogeneities; (c) creation of (barred) spiral galaxies as an indirect sign of space-time discreteness, recalling that detection of spirals in solid-state Physics is an indication of crystallization, galactic bars seen as topological defects between "helicoidal dislocations"; (d) Dark Matter interpreted as vacancies within regions with different structure than $L_{G}$, either of glass, amorphous (non-lattice) type, or like the Leech 
lattice or with just one $E_{8}$, without katoptrons or a $\mathrm{BEH}$ mechanism. In the latter case one would have DM "baryons" clamped in heavy dark objects analogous to neutron stars, to which usual baryogenesis arguments do not apply. Visible galaxies should be then considered as a kind of entropic droplets, crystal bubbles within a glass "mosaic" of DM (possibly amorphous) regions, similarly to quartz (silicon crystal) islands within silicon-glass. Estimating the ratio $R$ of crystal-to-glass-type volumes using Maxwell-Boltzmann statistics yields $R=1-\exp \left(-\Delta F /\left(k_{B} T_{\text {eff }}\right)\right), \Delta F$ measuring the free-energy difference between states and $T_{\text {eff }}$ a suitable temperature. A future analogue of Bragg crystallography might test the validity of this picture; (e) order-of-magnitude estimate of the total number of elementary particles (recall that related rudimentary scientific estimates date back to Archimedes' heliocentric model in "Psammites"), seen as defects with total entropy $S_{p}$ corresponding to an enthalpy cost $H$, as a function of the entropy $S_{B}$ of the Universe determined by its size, i.e. $S_{p}=S_{B} \exp \left(-H /\left(k_{B} T_{e f f}\right)\right)$; (f) a solution to the black-hole $(\mathrm{BH})$ information paradox, since here a BH is literally a "hole" within the space-time fabric gradually shrinking by Hawking radiation. Reconstruction of this fabric needs a precise number and structure of links between nodes, indicating that the information of each defect (particle) participating in the formation of a $\mathrm{BH}$ is stored in its surface topology, which in parallel connects the BH entropy with its area.

\subsection{Emergence of time, space, gravity and gauge symmetry}

We now replace $s_{i}$ with Grassmann variables $\Psi_{i}$ coupled via $\mathcal{E}_{i j}$ reflecting node connectivity in discretized spinor-gravity[26] inspired by Akama's spinor pre-geometry [27], assumed to belong to the $q=1$ Potts-model universality class. The non-compressibility (i.e.non-commutativity) of fermions maintains the lattice's discreteness, explaining the Heisenberg-uncertainty and Pauli-exclusion principles. Embedding the complexified $L_{G}$ in complex Euclidean space suitable for longer wavelengths (lower momenta $p$ ) endows it with an isometry $G(\mathbb{C})$, yielding an action, starting from $S_{l a t}=\sum_{<i, j>} \mathcal{E}_{i j} \bar{\Psi}_{i} \Psi_{j}$, over a compact toric Kähler manifold $T_{G(\mathbb{C})}^{d} \equiv \mathbb{C}^{d} / L_{G(\mathbb{C})}$ possessing a complex Liegroup structure:

$S_{f}(\Psi)=\int_{T_{G(\mathbb{C})}^{d}} d^{d} x \operatorname{det}\left(\frac{i}{2} \bar{\Psi} \gamma^{m} \partial_{\mu} \Psi+\right.$ h.c. $) \equiv \int_{T_{G(\mathbb{C})}^{d}} d^{d} x \operatorname{det}\left(\tilde{E}_{\mu}^{m}\right)=\int_{T_{G(\mathbb{C})}^{d}} d^{d} x \operatorname{det}\left(E_{\mu}^{m}\right) \sum_{N=0}^{\infty} \frac{\left[\sum_{M=1}^{\infty} \frac{(-1)^{M+1} \operatorname{tr}\left(\left(\left(E^{-1} \tilde{E}_{v}^{n}-\delta_{v}^{n}\right)^{M}\right)\right.}{M}\right]^{N}}{N !}$

using Jacobi's formula, with $d=16$ complex dimensions, h.c. standing for "hermitian conjugate", $\gamma^{m}$ appropriate Dirac matrices, $E_{\mu}^{m}$ the elements of a suitable matrix $E$, lower-case Greek and Latin indices correspond to space-time and to the "internal" Lorentz symmetry, $\tilde{N} \equiv M N \leq d$ due to the CayleyHamilton theorem, and integration over the torus $T_{G(\mathbb{C})}^{d}$ implies a UV cut-off $M_{\mathrm{Pl}} \sim L_{\text {Planck }}^{-1}$ due to a minimal distance between nodes. The variables $\bar{\Psi}_{i}, \Psi_{j}$ correspond in a dual sense to lattice vacancies and consist of two Weyl spinors of opposite chirality following the Poincaré-Hopf index theorem, transforming like $(\mathbf{2 4 8 , 1})$ (SM fermions) and $(\mathbf{1 , 2 4 8})$ (katoptrons) under $E_{8} \times E_{8}^{\prime}$. The conceptual advantage of the above consists in transferring the original non-commutativity of space-time nodes to the fields propagating in it so as to perform integrations and regularization preserving the symmetries. Defining a generating functional $W(J) \equiv \ln \left(\int \mathcal{D} \Psi \exp \left(\tilde{S}_{J}-S_{f}\right)\right)=S_{J}-\Gamma_{e f f}(E)$ with $\left\{\begin{array}{l}\tilde{S}_{J} \\ S_{J}\end{array}\right\}=\int d^{d} x J_{m}^{\mu}\left\{\begin{array}{l}\tilde{E}_{\mu}^{m} \\ E_{\mu}^{m}\end{array}\right\}$ for a source $J_{m}^{\mu}$, and $\Gamma_{e f f}(E)$ the effective action after a Legendre transformation, we choose the matrix $E_{\mu}^{m}=\frac{\delta W(J)}{\delta J_{m}^{\mu}} \equiv<\tilde{E}_{\mu}^{m}>\sim \delta_{\mu}^{m} M_{\mathrm{Pl}}$ for $\mu, m=0, \ldots, 3$ (0 otherwise), with elements vacuum-expectation values ("vevs") of composite bosons in a mean-field approximation. Further symmetry breaking of $G \rightarrow H \equiv E_{7} \times S L(2, \mathbb{C}) \times E_{7}^{\prime} \times S L(2, \mathbb{C})^{\prime}$ via vevs of antisymmetric bi-fermion operators transforming like 
$\mathbf{2 4 8} \times \mathbf{2 4 8} \rightarrow \mathbf{2 4 8}$ a under each of the $2 E_{8}$ s yields a 4 real- $d$ action $S_{e f f}\left(E_{\mu}^{m}, \psi, A_{\mu}, \phi_{\text {infl }}\right)$ with EinsteinHilbert and Yang-Mills terms, leading to the usual least-action-principle Euler-Lagrange equations in a lower order Taylor expansion for fluctuations $\delta E_{\mu}^{m} \ll E_{\mu}^{m}$ and for $\tilde{E}_{\mu}^{m}-E_{\mu}^{m} \equiv \bar{\psi} \gamma^{m} \partial_{\mu} \psi \ll E_{\mu}^{m}$ :

$$
S_{e f f} \sim \int_{\epsilon} d^{4} x \sqrt{\operatorname{det}\left(-g_{\mu \nu}(E)\right)}\left(k R(E)+k^{\prime} \operatorname{tr}\left(\left[D_{\mu}(A), D_{v}(A)\right]^{2}\right)+\bar{\psi} \gamma^{\mu}\left(D_{\mu}(A)+\delta_{\mu}(E)\right) \psi+\mathcal{L}\left(\phi_{\text {infl }}\right)+\ldots\right),
$$

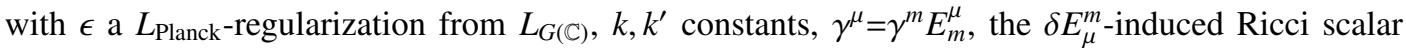
$R(E)$ (omitting small terms consistent with present gravitational tests) and derivative correction $\delta_{\mu}(E) \equiv\left(E_{m}^{v} \partial_{\mu}\left(E_{v}^{m}\right)+E_{\mu}^{m} \partial_{v}\left(E_{m}^{v}\right)\right) / 2$, and $D_{\mu}(A)$ the covariant derivative of the $E_{7} \times E_{7}^{\prime}$ Kaluza-Klein compactification gauge fields $A_{\mu}$. The metric is $g_{\mu \nu}(E)=E_{\mu}^{m}(x) E_{\nu}^{n}(x) \eta_{m n}$, with $\eta_{m n}=\operatorname{diag}(-1,1,1,1)$ Minkowski's space-time as the $S L(2, \mathbb{C})$ subgroups of the two $E_{8}$ s break to their diagonal subgroup $S O(1,3)_{D}$ due to $M_{P l}$-scale vevs of $2 \tilde{N}$-fermion operators $\left(O\left(p / M_{\mathrm{Pl}}\right)^{\tilde{N}}\right.$ after Fourier transformation) for $\tilde{N}=2$ in Eq.(11) coupling the left- and right-handed sectors and transforming like $(\mathbf{1 , 3}, \mathbf{1 , 3})$ under $H$. At slightly lower energies, after the self-breaking only of the SM-fermion $S U(3)$ family group due to a parity-violating $L_{G}$ asymmetry, similar operators transforming like $(\mathbf{2 4 , 2 4})$ under $\tilde{H} \equiv S U(5) \times S U(5)^{\prime} \subset E_{7} \times E_{7}^{\prime} \subset H$ lead to $\tilde{H} \rightarrow S U(5)_{D}$, obviating the need for both outer automorphisms in [13]. Moreover, $\mathcal{L}\left(\phi_{\text {infl }}\right)$ is a Lagrangian for inflatons as compactified gauge-field components, including scalar kinetic terms, a "sombrero" potential [13] and the cosmological constant, generating entropy and a time arrow, diluting the 't Hooft-Polyakov monopole primordial abundance and explaining the Universe's 4- $d$ expansion, isotropy, homogeneity and flatness, which in parallel reinforces the validity of our approximation.

The action above leads to quantized gravitational equations $\frac{\delta \Gamma_{e f f}(E)}{d E_{m}^{\mu}}=J_{\mu}^{m}$, with the metric and the gauge fields induced by composite-boson operators. Further symmetry breaking takes place starting from a unified critical coupling at $M_{\mathrm{Pl}}$, estimated via Schwinger-Dyson equations as $\alpha\left(\mathrm{M}_{\mathrm{Pl}}\right) \sim 1 / C_{2}\left(E_{8}\right) \sim 0.03$, down to the SM having the known fermion-family structure with a non-perturbative BEH mechanism based on katoptrons at $\Lambda_{K} \sim M_{\mathrm{Pl}} \exp \left(-1.23 C_{2}\left(E_{8}\right)\right) \sim 1 \mathrm{TeV}$ [13] in a way similar to QCD asymptotic freedom [28], followed by a corresponding mirror mesonic spectrum.

\section{Conclusions}

Our purpose has been to link potential discoveries at the LHC with theories having implications on cosmology and on our understanding of space-time. Theories accommodating forthcoming experimental data might be found to be consistent, if not indicative, either of an extended nature of elementary particles or of a certain discreteness of space-time which should not be treated as background but as emergent, dynamical and non-perturbative in a sense dual to elementary particles. Regarding the mirror world, we sketched the conceptual path leading from the optimal connectivity principle to strongly-interacting mirror fermions and to the experimental detection of their mesonic bound states. Distinguishing the relevant signals from alternative models should focus our attention on distinctive processes related to particles such as lepto-quarks and perform relevant computer simulations.

\section{Acknowledgements}

Particular thanks are due to T. Papadopoulou for her support and encouragement, and to the organizers of an excellent ICFP2015 in the OAC (Kolymbari). This research has been co-financed in part by the E. U. (European Social Fund-ESF) and Greek national funds through the Operational Program "Education and Lifelong Learning" of the National Strategic Reference Framework (NSRF) - Research Funding Program: THALES. Investing in knowledge society through the European Social Fund. 


\section{References}

[1] N. Arkani-Hamed, S. Dimopoulos, G. Dvali, Phys. Lett. B429 (1998) 263; I. Antoniadis et. al., Phys. Lett. B436 (1998) 257; E. G. Floratos, G. K. Leontaris, Phys. Lett. B465 (1999) 95.

[2] S. Giddings, S. Thomas, Phys. Rev. D65 (2002) 056010; T. Rizzo, Phys. Rev. D60 (1999) 075001.

[3] ATLAS coll., Phys. Rev. D87 (2013) 1, 015010; CMS coll., arXiv:1408.3583 [hep-ex].

[4] S. Dimopoulos, H. Georgi, Nucl. Phys. B193 (1981) 150.

[5] M. Green, J. Schwarz, E. Witten, Superstring theory, Vol. 1,2 (2012), Cambridge University Press.

[6] M. Axenides, E. G. Floratos, L. Perivolaropoulos, Phys. Rev. D66 (2002) 085006.

[7] ATLAS coll., arXiv:1508.06608 [hep-ex]; CMS coll., JHEP 04 (2015) 124.

[8] T. D. Lee, C. N. Yang, Phys. Rev. 104 (1956) 254.

[9] F. Wilczek, A. Zee, Phys. Rev. D25 (1982) 553.

[10] S. Weinberg, Phys. Rev. D13 (1976) 974; L. Susskind, Phys. Rev. D20 (1979) 2619.

[11] J. Bardeen, L. N. Cooper, J. R. Schrieffer, Phys. Rev. 106 (1962) 162.

[12] G. Triantaphyllou, Eur. Phys. J. C10 (1999) 703.

[13] G. Triantaphyllou, El. J. Th. Phys. 10 (2013) 135; ibid., Eur. Phys. J. Web Conf. 70 (2014) 00051.

[14] J. C. Pati, A. Salam, Phys. Rev. $D 8$ (1973) 1240; H. Georgi, S. L. Glashow, Phys. Rev. Lett. 32 (1974) 438.

[15] E. Eichten, I. Hinchliffe, K. Lane, C. Quigg, Rev. Mod. Phys. 56 (1984) 579.

[16] G. Triantaphyllou, Mod. Phys. Lett. A16 (2001) 53.

[17] G. Triantaphyllou, J. Phys. G26 (2000) 99.

[18] G. Triantaphyllou, Int. J. Mod. Phys. A15 (2000) 265.

[19] E. Fahri, L. Susskind, Phys. Rep. 74 (1981) 277.

[20] T. Appelquist, G. Triantaphyllou, Phys. Rev. Lett. 69 (1992) 2750.

[21] ATLAS coll., arXiv:1506.00962 [hep-ex]; CMS Collaboration, JHEP 1408 (2014) 173.

[22] K.A. Olive et al. (Particle Data Group), Chin. Phys. C38 (2014) 090001.

[23] P. Jizba, H. Kleinert, F. Scardigli, Phys. Rev. D81 (2010) 084030.

[24] P. Sikivie, F. Gürsey, Phys. Rev. D16 (1977) 816; I. Bars, M. Gunaydin, Phys. Rev. Lett. 45 (1980) 859; N. S. Baaklini, Phys. Lett. B91 (1980) 376.

[25] G. A. Kabatianskii, V. I. Levenshtein, Probl. Inform. Transm. 14, 1 (1978) 1.

[26] A. Hebecker, C. Wetterich, Phys. Lett. B574 (2003) 269.

[27] K. Akama, Prog. Th. Ph. 60 (1978) 1900; D. Amati, G. Veneziano, Nucl. Phys. B204 (1982) 451.

[28] D. J. Gross, F. Wilczek, Ph.Rev.Lett. 30 (1973), 1343; H. D. Politzer, Ph.Rev.Lett. 30 (1973), 1346. 